01.5

\title{
Новый подход к экспериментальному исследованию больших ансамблей радиотехнических генераторов со сложными связями
}

\author{
(ㄷ Д.Д. Кульминский ${ }^{1,2}$, В.И. Пономаренко ${ }^{1,2}$, И.В. Сысоев ${ }^{1,2}$, М.Д. Прохоров ${ }^{1}$ \\ ${ }^{1}$ Саратовский филиал Института радиотехники и электроники им. В.А. Котельникова РАН, Саратов, Россия \\ ${ }^{2}$ Саратовский национальный исследовательский государственный университет им. Н.Г. Чернышевского, Саратов, Россия \\ E-mail: kulminskydd@gmail.com
}

Поступило в Редакцию 22 августа 2019г.

В окончательной редакции 22 августа 2019r.

Принято к публикации 14 ноября 2019 г.

\begin{abstract}
Предложен новый подход, позволяющий экспериментально исследовать сложную динамику в больших ансамблях связанных радиотехнических генераторов. Подход применен для построения аналого-цифровой экспериментальной установки для исследования ансамблей генераторов с запаздывающей обратной связью, в которой реализована возможность задания произвольной архитектуры связей и разных типов связей между генераторами. Продемонстрирована возможность реконструкции сложной топологии связей и параметров всех генераторов по их экспериментальным временны́м рядам.
\end{abstract}

Ключевые слова: ансамбли осцилляторов, радиофизический эксперимент, генераторы с запаздыванием, реконструкция уравнений.

DOI: 10.21883/PJTF.2020.04.49046.18018

Задача исследования сложной динамики ансамблей связанных осцилляторов уже давно привлекает к себе внимание многих авторов [1-6]. В большинстве случаев для исследования больших ансамблей осцилляторов применялись теоретические и численные методы, а экспериментальным исследованиям уделялось значительно меньше внимания. Это объясняется сложностью постановки натурного эксперимента, которая быстро возрастает с увеличением размера ансамбля и количества связей между его элементами. Удобными объектами для экспериментального изучения сложной динамики ансамблей осцилляторов являются радиотехнические генераторы. С их помощью были исследованы процессы синхронизации в цепочках связанных осцилляторов $[7,8]$ и в ансамблях осцилляторов, связанных через общее поле $[9,10]$. Однако экспериментальная реализация сложной архитектуры связей и сложных функций связи между радиотехническими генераторами в больших ансамблях остается пока трудной задачей.

В настоящей работе предложен новый подход к экспериментальному исследованию больших ансамблей радиотехнических генераторов со сложными связями, в основе которого лежит программный способ формирования сигналов, отвечающих за связь между генераторами. Такой подход позволяет задать произвольную архитектуру связей и реализовать практически любой вид связей между генераторами в радиофизическом эксперименте.

Для экспериментального исследования больших ансамблей радиотехнических генераторов нами построена оригинальная аналого-цифровая установка, блок-схема которой представлена на рис. 1, $a$. Для осуществления связи между генераторами была использована система ввода-вывода многоканальных данных - National
Instruments PCI eXtensions for Instrumentation (NI PXI). Сигналы напряжения $V_{i}(t)$ с выхода каждого генератора (где $i=1, \ldots, N ; N-$ число генераторов) подаются на аналоговые входы многоканального аналогоцифрового преобразователя и оцифровываются с частотой $f=10 \mathrm{kHz}$ для дальнейшей обработки. Затем с помощью программы на LabView проводится преобразование сигналов $V_{i}(t)$ и формируются отвечающие за связь генераторов сигналы

$$
K_{i}(t)=\sum_{j=1}^{N} k_{i, j}\left(V_{j}(t)-V_{i}(t)\right),
$$

где $k_{i, j}$ - коэффициенты, характеризующие силу связи. Сигналы $K_{i}(t)$ преобразуются в аналоговый вид с помощью многоканального цифро-аналогового преобразователя и подаются в качестве управляющего воздействия на вход каждого генератора.

В рамках настоящей работы в установке реализована простая линейная связь между генераторами. При аналоговом моделировании такая связь соответствует связи двух радиотехнических генераторов через резистор [11]. Помимо резисторов для связи генераторов можно использовать и другие элементы: конденсатор, катушку индуктивности или мемристор, реализуя емкостную [12], индукционную [13] или мемристорную связь $[14,15]$ соответственно. Поскольку сигналы, отвечающие за связь генераторов, формируются в экспериментальной установке программным образом, в ней можно реализовать связь генераторов через резистор, конденсатор, катушку индуктивности или мемристор, внеся соответствующие изменения в программу. Кроме того, можно легко задать произвольную архитектуру связей между генераторами и реализовать различные 

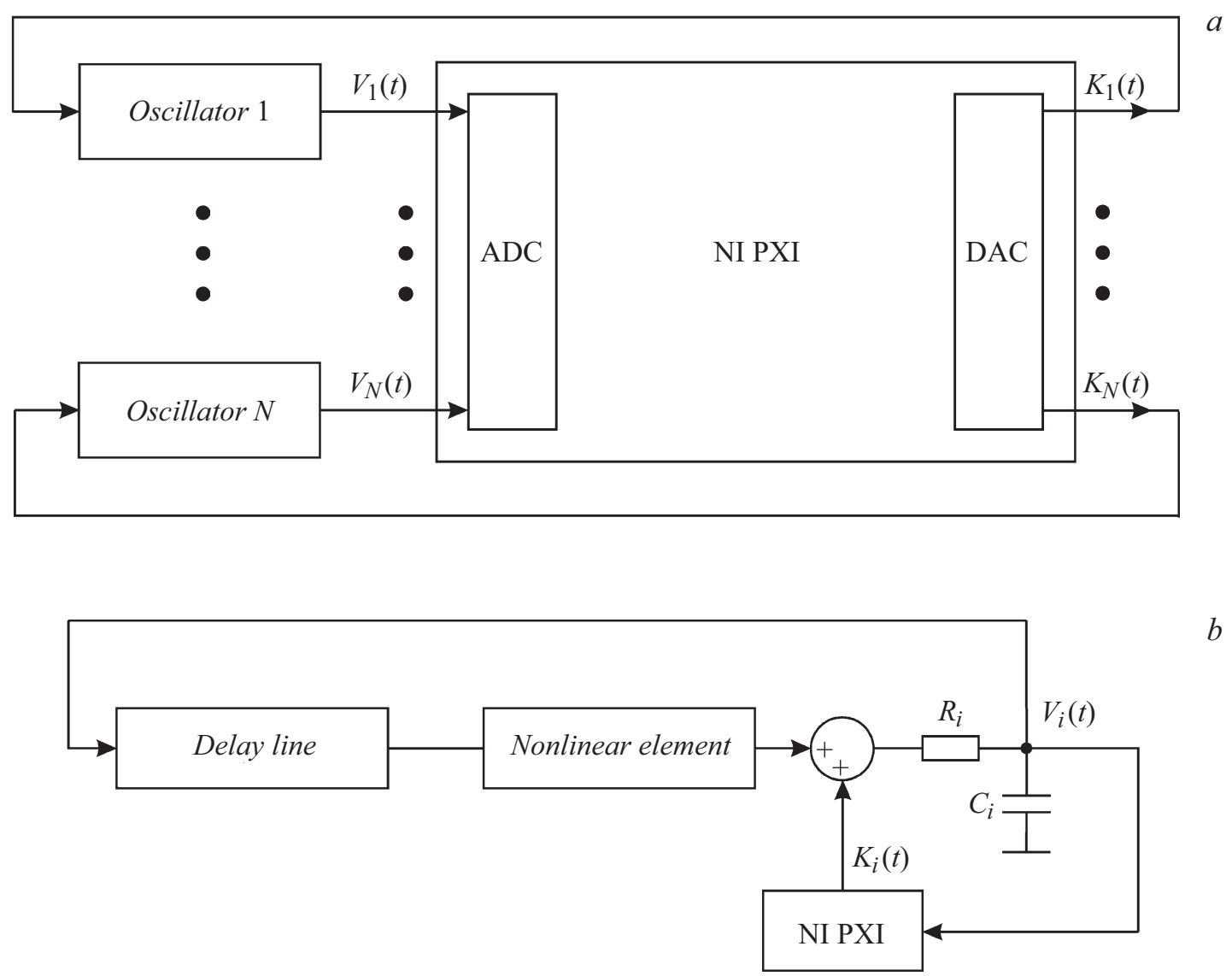

Рис. 1. $a-$ блок-схема экспериментальной установки. Показаны только первый и $N$-й генераторы. NI PXI - система ввода-вывода многоканальных данных, ADC - аналого-цифровой преобразователь, DАC - цифро-аналоговый преобразователь. $b-$ блок-схема базового генератора.

виды нелинейных и запаздывающих связей. Установка работает в режиме реального времени и позволяет при необходимости подстраивать в ходе эксперимента силу связей для управления коллективной динамикой генераторов.

В качестве базового элемента ансамбля мы выбрали генератор с запаздывающей обратной связью, представляющий собой кольцевую систему, состоящую из линии задержки, нелинейного элемента и низкочастотного $R C$-фильтра первого порядка. Блок-схема базового генератора представлена на рис. $1, b$. Связь базового генератора с другими генераторами ансамбля реализована подачей сигнала связи $K_{i}(t)$ на вход сумматора, расположенного между фильтром и нелинейным элементом. Динамика $i$-го генератора с запаздыванием описывается модельным уравнением следующего вида:

$$
R_{i} C_{i} \dot{V}_{i}(t)=-V_{i}(t)+f_{i}\left(V_{i}\left(t-\tau_{i}\right)\right)+K_{i}(t),
$$

где $V_{i}(t)$ и $V_{i}\left(t-\tau_{i}\right)$ - напряжения на входе и выходе линии задержки соответственно, $\tau_{i}$ - время запаздывания, $R_{i}$ и $C_{i}$ - сопротивление и емкость элементов фильтра, $f_{i}$ - передаточная характеристика нелинейного элемента, а $K_{i}(t)$ имеет вид (1). В качестве базового осциллятора ансамбля можно использовать также другие автогенераторы. Отметим, что похожий подход был использован нами для экспериментального исследования синхронизации двух генераторов, описываемых уравнениями ФитцХью-Нагумо, взаимно связанных запаздывающей связью [16].

Для иллюстрации работоспособности созданной экспериментальной установки мы провели реконструкцию модельных уравнений (2) ансамбля, состоящего из восьми связанных генераторов с запаздыванием. Из 56 возможных связей в ансамбле случайным образом задано 16 однонаправленных связей. Все генераторы ансамбля являются неидентичными. Их параметры принимают случайные значения в следующих интервалах: $\tau_{i} \in[25,42.5] \mathrm{ms}, R_{i} C_{i} \in[2,4.8] \mathrm{ms}$, $k_{i, j} \in[0.05,0.15]$. Все нелинейные элементы генераторов имеют квадратичную передаточную характеристику. На рис. 2, $a$ приведен фрагмент временно́й реализации сигнала $V_{1}(t)$ в первом генераторе, имеющем параметры $\tau_{1}=25 \mathrm{~ms}, R_{1} C_{1}=2 \mathrm{~ms}, k_{1,4}=0.15, k_{1,8}=0.08, k_{1, j}=0$ при $j \neq 4,8$.

Используя предложенный нами ранее метод реконструкции [17], основанный на минимизации для каждого генератора целевой функции, характеризующей рас- 

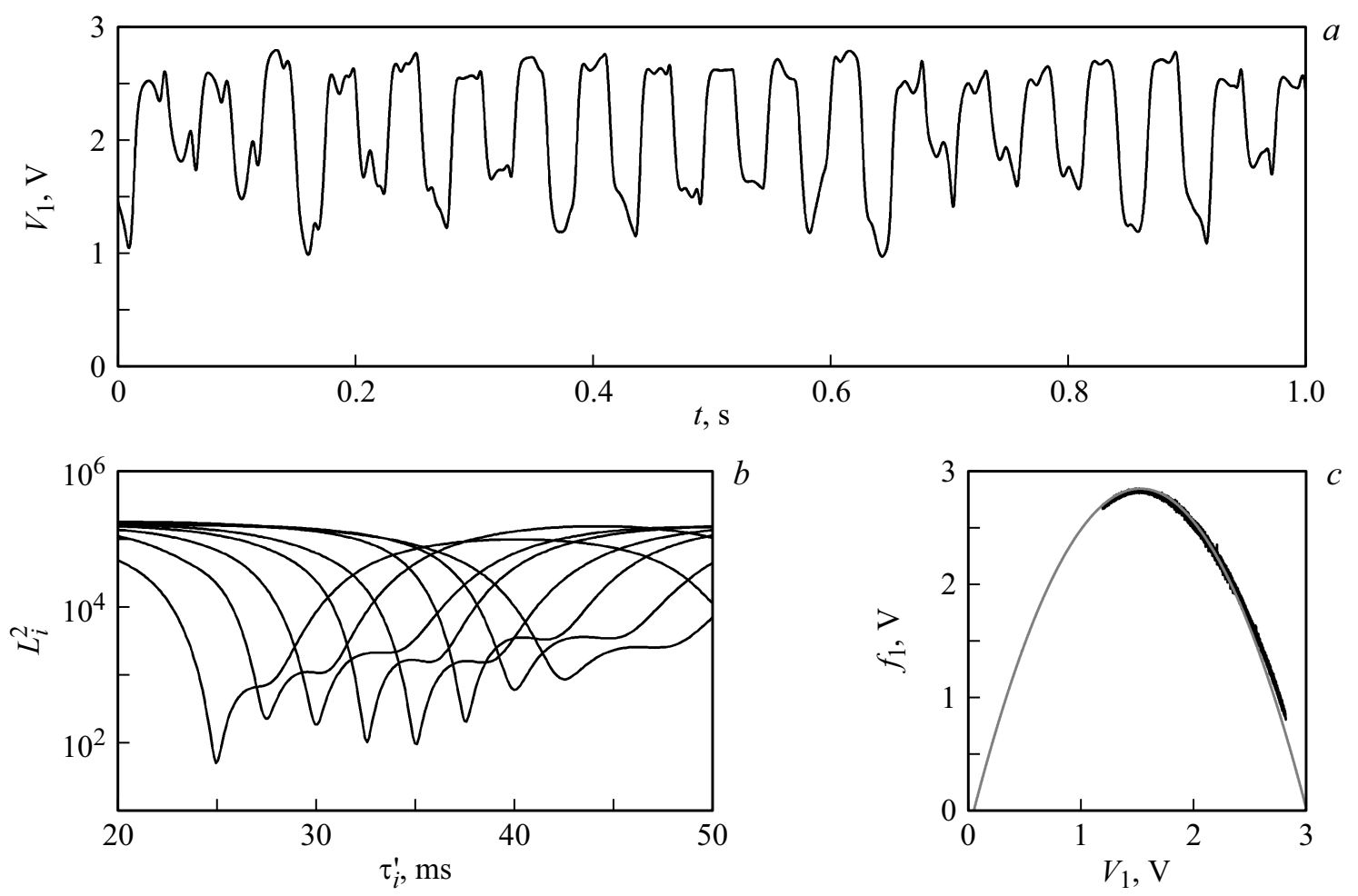

Рис. 2. $a$ - временна́я реализация сигнала $V_{1}(t)$ первого генератора; $b-$ зависимости $L_{i}^{2}\left(\tau_{i}^{\prime}\right)$ для каждого из восьми генераторов ансамбля; $c$ - восстановленная нелинейная функция (жирная линия) и истинная функция $f_{1}$ (светлая линия) первого генератора.

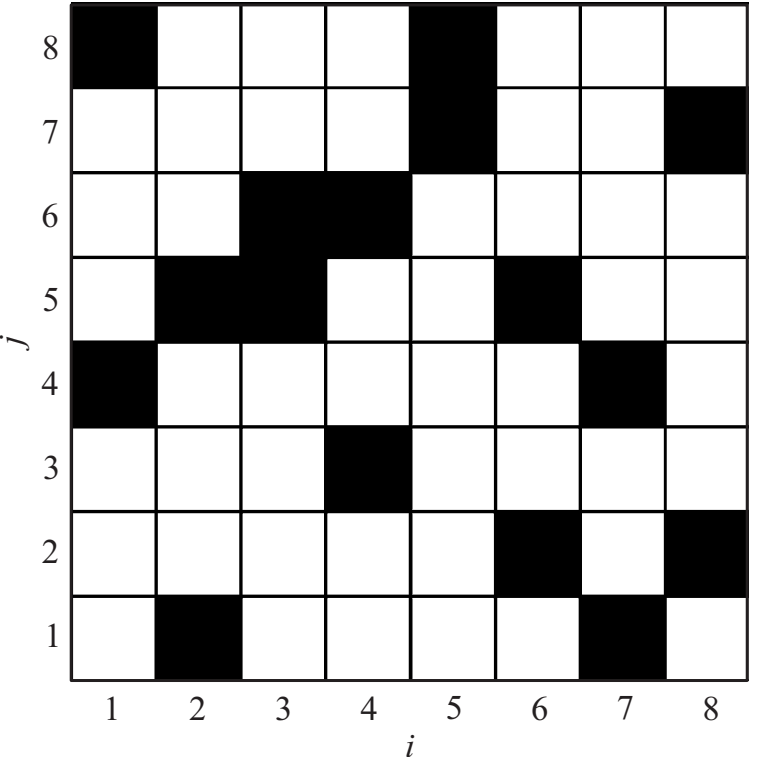

Pис. 3. Диаграмма результата реконструкции архитектуры связей. Черным цветом показаны правильно восстановленные связи, белым - правильно диагностированные отсутствующие связи.

стояние между точками реконструируемой нелинейной функции, мы построили на рис. $2, b$ зависимости целевой функции $L_{i}^{2}$ от пробного времени запаздывания $\tau_{i}^{\prime}$ для всех восьми генераторов ансамбля. Глобальные миниму- мы пяти зависимостей $L_{i}^{2}\left(\tau_{i}^{\prime}\right)$, в том числе $L_{1}^{2}\left(\tau_{1}^{\prime}\right)$, наблюдаются при истинных временах запаздывания генераторов. Для трех генераторов минимум $L_{i}^{2}\left(\tau_{i}^{\prime}\right)$ оказался смещен относительно истинного времени запаздывания на время $t_{s}=0.1 \mathrm{~ms}$, равное интервалу выборки точек временны́х рядов.

Восстановленные коэффициенты связи для первого генератора $k_{1,4}^{\prime}=0.148$ и $k_{1,8}^{\prime}=0.079$ достаточно близки к истинным значениям. Восстановленный параметр инерционности $\left(R_{1} C_{1}\right)^{\prime}=2.1 \mathrm{~ms}$ тоже близок к истинному значению $R_{1} C_{1}$. Точность восстановления параметров остальных генераторов ансамбля получилась примерно такой же.

На рис. 2, с приведена нелинейная функция первого генератора, восстановленная при $k_{1,4}^{\prime}, k_{1,8}^{\prime}$ и $\left(R_{1} C_{1}\right)^{\prime}$. Она достаточно хорошо совпадает с истинной передаточной характеристикой $f_{1}$ нелинейного элемента первого генератора. Аналогичным образом мы восстановили нелинейные функции остальных генераторов, получив высокое качество реконструкции.

Результат реконструкции архитектуры связей в ансамбле приведен на рис. 3. Клетка с координатами $(i, j)$ показывает влияние $j$-го генератора на $i$-й, кроме клеток на диагонали, не несущих никакой информации. Метод позволил выявить все 16 имеющихся в ансамбле связей, ложных связей не обнаружено.

Итак, нами предложен перспективный подход к экспериментальному исследованию больших ансамблей радиотехнических генераторов с большим количеством 
связей. Используя предложенный подход, мы построили экспериментальную установку, которая позволяет программным образом задавать произвольную архитектуру и тип связей между генераторами и при необходимости изменять связи в ходе эксперимента. Работоспособность установки продемонстрирована на примере ансамбля генераторов с запаздывающей обратной связью. С ее помощью впервые проведена реконструкция сложной архитектуры связей, коэффициентов связей и собственных нелинейных функций генераторов с запаздыванием по их экспериментальным временны́м рядам.

\section{Финансирование работы}

Работа выполнена при поддержке гранта Президента РФ, проект МК-1199.2019.8 (дизайн экспериментальной установки), а также Российского фонда фундаментальных исследований, проект № 19-02-00071 (исследование и реконструкция ансамблей генераторов с запаздыванием).

\section{Конфликт интересов}

Авторы заявляют, что у них нет конфликта интересов.

\section{Список литературы}

[1] Afraimovich V.S., Nekorkin V.I., Osipov G.V., Shalfeev V.D. Stability, structures, and chaos in nonlinear synchronization networks. Singapore: World Scientific, 1995. 260 p. DOI: $10.1142 / 2412$

[2] Boccaletti S., Latora V., Moreno Y., Chavez M., Hwang D.-U. // Phys. Rep. 2006. V. 424. P. 175-308. DOI: 10.1016/j.physrep.2005.10.009

[3] Osipov G.V., Kurths J., Zhou C. Synchronization in oscillatory networks. Berlin: Springer, 2007. 370 p. DOI: $10.1007 / 978-3-540-71269-5$

[4] Масленников О.В., Некоркин В.И. // УФН. 2017. Т. 187. № 7. C. 745-756. DOI: $10.3367 /$ UFNr.2016.10.037902

[5] Kundu P., Sharma L., Nandan M., Ghosh D., Hens C., Pal P. // Chaos. 2019. V. 29. P. 013112. DOI: $10.1063 / 1.5051535$

[6] Голдобин Д.С., Долматова А.В. // Изв. вузов. Прикладная нелинейная динамика. 2019. Т. 27. В. 3. С. 33-60. DOI: 10.18500/0869-6632-2019-27-3-33-60

[7] Gambuzza L.V., Buscarino A., Chessari S., Fortuna L., Meucci R., Frasca M. // Phys. Rev. E. 2014. V. 90. P. 032905. DOI: 10.1103/PhysRevE.90.032905

[8] Arumugama E.M.E., Spano M.L. // Chaos. 2015. V. 25. P. 013107. DOI: 10.1063/1.4905856

[9] Temirbayev A.A., Nalibayev Y.D., Zhanabaev Z.Z., Ponomarenko V.I., Rosenblum M. // Phys. Rev. E. 2013. V. 87. P. 062917. DOI: 10.1103/PhysRevE.87.062917

[10] Ponomarenko V.I., Kulminskiy D.D., Prokhorov M.D. // Phys. Rev. E. 2017. V. 96. P. 022209.

DOI: 10.1103/PhysRevE.96.022209

[11] Sysoev I.V., Prokhorov M.D., Ponomarenko V.I., Bezruchko B.P. // Phys. Rev. E. 2014. V. 89. P. 062911. DOI: 10.1103/PhysRevE.89.062911
[12] Liu Z., Ma J., Zhang G., Zhang Y. // Appl. Math. Comput. 2019. V. 360. P. 94-106. DOI: 10.1016/j.amc.2019.05.004

[13] Yao Z., Ma J., Yao Y., Wang C. // Nonlinear Dynamics. 2019. V. 96. P. 205-217. DOI: 10.1007/s11071-019-04784-2

[14] Xи Y., Jia Y., Ma J., Alsaedi A., Ahmad B. // Chaos Soliton Fract. 2017. V. 104. P. 435-442. DOI: $10.1016 /$ j.chaos.2017.09.002

[15] Корнеев И.А., Шабалина О.Г., Семенов В.В., Вадивасова T.E. // Изв. вузов. Прикладная нелинейная динамика. 2018. T. 26. B. 2. C. 24-40. DOI: $10.18500 / 0869-6632-2018-26-2-24-40$

[16] Пономаренко В.И., Кульминский Д.Д., Прохоров М.Д. // Письма в ЖТФ. 2018. Т. 44. В. 17. С. 11-18. DOI: 10.21883/PJTF.2018.17.46565.17366

[17] Sysoev I.V., Ponomarenko V.I., Kulminskiy D.D., Prokhorov M.D. // Phys. Rev. E. 2016. V. 94. P. 052207. DOI: 10.1103/PhysRevE.94.052207 